\title{
A Force Based Model of Individual Cell Migration with Discrete Attachment Sites and Random Switching Terms
}

\author{
J. C. Dallon \\ dallon@math.byu.edu \\ Matthew Scott \\ Brigham Young University \\ William V. Smith \\ Brigham Young University
}

Follow this and additional works at: https://scholarsarchive.byu.edu/facpub

Part of the Mathematics Commons

\section{Original Publication Citation}

Journal of Biomechanical Engineering 135(7):71008. (2013) doi: 10.1115/1.4023987

\section{BYU ScholarsArchive Citation}

Dallon, J. C.; Scott, Matthew; and Smith, William V., "A Force Based Model of Individual Cell Migration with Discrete Attachment Sites and Random Switching Terms" (2013). Faculty Publications. 2719.

https://scholarsarchive.byu.edu/facpub/2719 
To appear in Journal of Biomechanical Engineering

\title{
A FORCE BASED MODEL OF INDIVIDUAL CELL MIGRATION WITH DISCRETE ATTACHMENT SITES AND RANDOM SWITCHING TERMS
}

\author{
J. C. Dallon* \\ Matthew Scott \\ W. V. Smith \\ Department of Mathematics, \\ Brigham Young University, \\ Provo, UT
}

\begin{abstract}
A force based model of cell migration is presented which gives new insight into the importance of the dynamics of cell binding to the substrate. The main features of the model are the focus on discrete attachment dynamics, the treatment of the cellular forces as springs, and an incorporation of the stochastic nature of the attachment sites. One goal of the model is to capture the effect of the random binding and unbinding of cell attachments on global cell motion. Simulations reveal one of the most important factor influencing cell speed is the duration of the attachment to the substrate. The model captures the correct velocity and force relationships for several cell types.
\end{abstract}

\section{Introduction}

Individual cell motion is fundamental to many systems. There are several ways cells can move. They may passively move when the surrounding material transports them as occurs in the bloodstream. They may actively move with the aid of cilia, flagella, or they can move by changing their cell shape and attach appendages called pseudopods to the surrounding material. It is this last type of cell motion called amoeboidal cell motion which is the focus of this paper. Important examples of amoeboidal cell motion include white blood cells, fibroblasts, endothelial cells, epithelial cells, and finally Dictyostelium discoideum (Dd).

Cell motion is fundamental to many biological processes including wound healing, cancer, and morphogenesis. When a wound in the skin heals, various cell types are recruited to the wound region. These include leukocytes, macrophages and fibroblasts migrating from the surrounding tissue to the wound region [1]. In addition the epidermis is closed as epidermal cells migrate into the wound region restoring the integrity of the epidermis [2,3]. Similarly cancer cell aggregation, growth, and motion is critical to understanding cancer and thereby determining treatments and outcomes. Thus if a cancer tumor remains cohesive and malignant cells do not migrate into surrounding tissue, there are fewer complications and the overall outcome for the patient is typically better [4,5]. Cell interaction is vital to morphogenetic processes. For example in embryonic development, the collective motion of a mass of cells alters the shape of the mass via extension and conversion, whereby cells interdigitate to narrow and lengthen tissue [6]. This cell motion not only changes the shape of the early embryo but localizes cells and alters the physical properties of the extracellular matrix in preparation for cell differentiation [7]. Cell aggregation is also important in the life cycle of the amoeba Dictyostelium discoideum (Dd). In Dd, after a period of starvation individual amoebae become chemotactically sensitive and aggregate towards cells which periodically emit a chemoattractant. Once cells have aggregated, they form a slug composed of several thousand individual ameobae. Acting in a coordinated manner, the individual cells cause the slug to move [8].

*Address all correspondence to this author. dallon@math.byu.edu

Feb 2013 


\section{Methods}

\subsection{Background}

2.1.1 Amoeboidal Cell Motion Adhesion serves two major functions in migration. It generates traction by linking the extracellular substratum to actomyosin filaments, and it organizes the signaling networks that regulate migration and other cellular processes including proliferation, gene expression, and cell survival $[9,10]$. The linkage between the extracellular matrix and actin has been known for over 30 years based on electron microscopic and fluorescence colocalization studies [11-13]. The mechanosensing and mechanotransduction that occurs in cell migration is not well understood and is an area of active research.

As cells move along a substrate or extracellular matrix, in general they do so by extending an appendage called a pseudopod via actin filament polymerization. These pseudopods attach to the substrate via integrin receptors which cluster to form nascent adhesions. These nascent adhesions progress to focal complexes which can become focal adhesions and then fibrillar adhesions [14]. A contractile force is generated and this moves the cell body. The integrin mediated adhesions are then disassembled as they arrive at the trailing end of the cell where the adhesion complexes can be torn out of the cell membrane and left behind in the extracellular matrix [15].

2.1.2 Cell Migration Modeling The main merits of the model presented here are the focus on discrete attachment site dynamics, the treatment of the cellular forces, and an incorporation of the attachments (or binding sites) all with the purpose of investigating whole cell migration. The modeling of discrete attachment site dynamics is not a detailed biochemical treatment; it focuses on a phenomenological treatment at the focal adhesion level. The main intent is to capture the affect of the random binding and unbinding of cell attachments on global cell motion while achieving tractable model complexity.

There are many mathematical models of cell migration which can be classified in many ways. One convenient classification involves three space-time scales: an intracellular scale, a whole cell scale, and a multi-cellular scale [16]. Of these models there are some in each of the categories which have traits in common with the model presented here.

The models which focus in detail on the subcellular scale include those which model different subcellular regions of the cell with viscoelastic elements like Sandersius and Newman [17]. The focus of their modeling is to capture whole cell behavior by linking subcellular forces. Other models focus on subcellular processes like actin polymerization [18-20] or integrin attachments [21] which are essential for cell migration. Schreiber et. al. [20] investigate cell forces-velocity relationships but not at the whole cell level and their modeling is very different from ours. Paszek et. al. [21] model the integrin attachment process in a detailed biochemical manner using force terms with a Hookean law to help explain integrin clustering. Although there are similar details in their modeling compared to ours, the focus is entirely different and at a different scale.

There are several whole cell models. Of the four mentioned here, the first three are force based. Of the force based models two model the cytoskeletal structure and the actin polymerization forces in a phenomenological way. One uses the immersed boundary method [22] and the other uses conservation equations, which consider the network drag, pressure, and stress to determine equations representing the flow of the network and the motion of the cell [23]. The final force based model focuses on attachments as does the model presented here [24]. They also use Hookean laws to determine forces and cell motion but the model formulation is quite different and does not focus on the stochastic nature of the binding and unbinding events. The last whole cell model is one that models motion with stochastic ordinary differential equations using white noise [25]. It does consider the stochastic nature of the cell motion but in a very different formulation than the way it is modeled in this paper.

Finally we mention two multi-cell force based models. The first uses viscoelastic forces for the cell but does not treat the binding site attachment forces in a stochastic or discrete manner [26]. The cell is treated as the fundamental object. The other model does focus on attachments in a phenomenological manner with a continuum description of integrin based adhesions [27].

\subsection{The Model}

Our model emphasizes the forces involved in cell motion with a simplified approach to the way cells attach to the substrate. A cell is modeled as several interaction sites representing adhesion sites [28,29]. Sites from the cell exert force on a common location, the nucleus (see figure 1). Binding sites interact with a substrate by attaching to it. When a binding site attaches to the substrate it remains fixed at that substrate location. The adhesion sites are reinforced by the cell to become more stable allowing them to persist for a greater duration and exert greater forces. It is likely that the duration time of the adhesion is related to the force applied to the adhesion. Certainly nascent adhesions with too much force will not persist, whereas focal adhesions can persist with greater forces but with less force they may start to disassemble. Initially it will be assumed that the duration of the interaction of the adhesion site is independent of the force on the site. In the case of Dd where actin foci are believed to be related to the attachment sites, the duration of the actin foci has been measured [30] (see figure 2). The mechanisms resulting in cell motion may vary depending on the cell type but at the level of our mathematical model the description is the same, i.e., randomly adhering sites which exert forces on the cell and the substrate. 

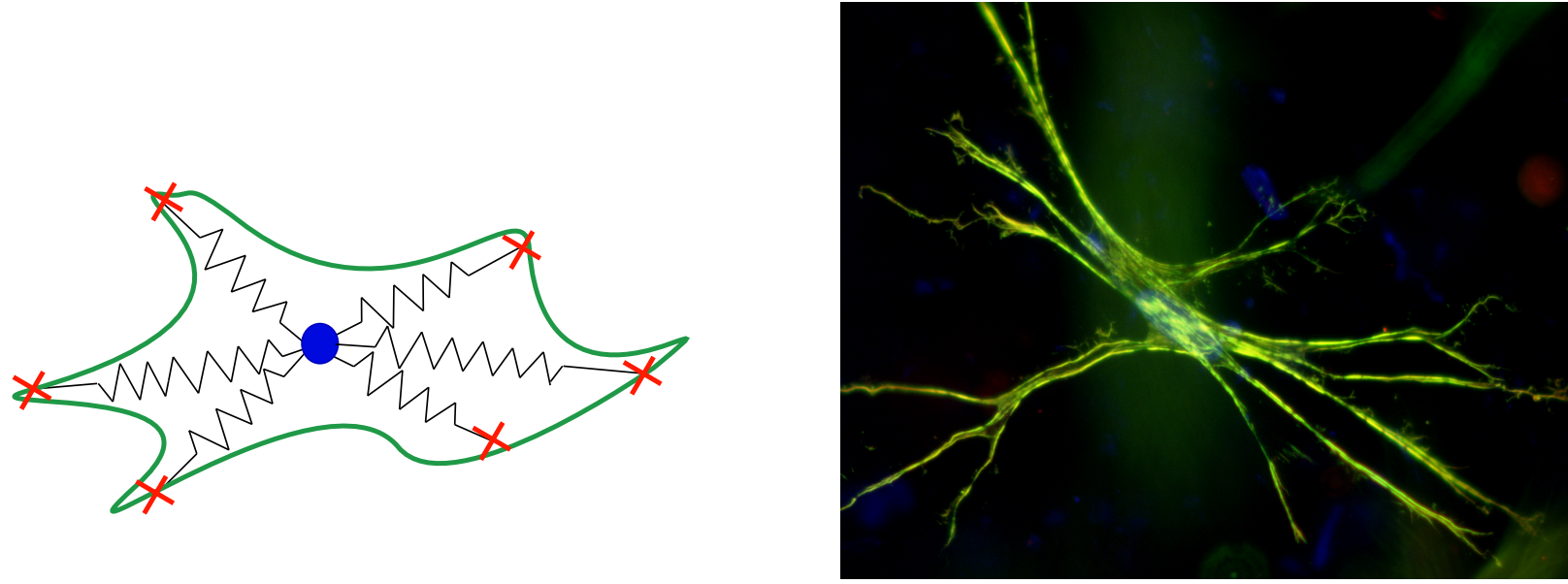

FIGURE 1. The left panel depicts the way we model a cell mathematically. The cell is a center location (nucleus) with attached springs. The other ends of the springs are attached to sites which can interact with the extracellular matrix (membrane bound attachment sites) depicted by " $x$ ". The right panel is an image of a fibroblast in a collagen lattice.

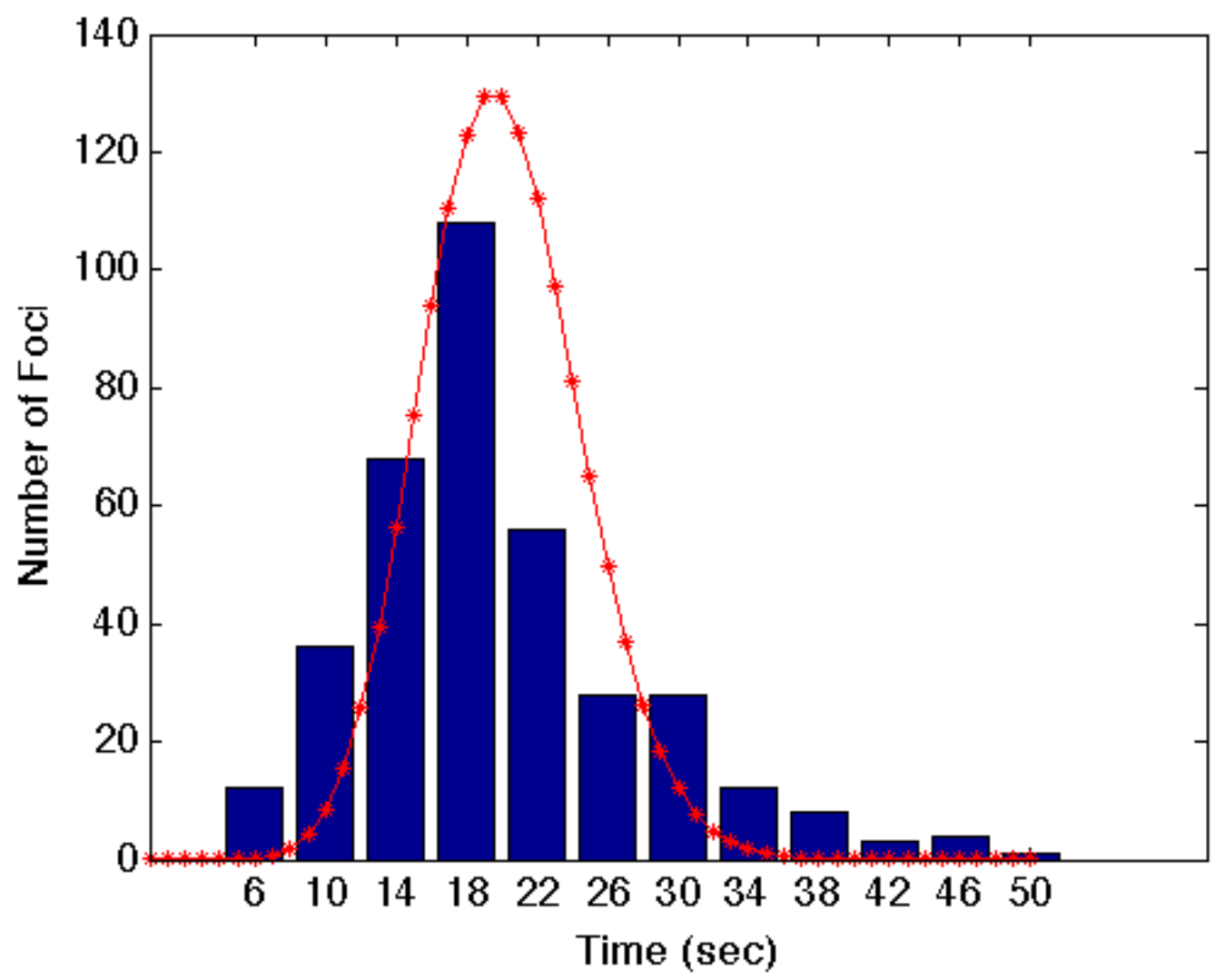

FIGURE 2. Data for the duration time of actin foci from wild type Dd cells is plotted as bar graphs [30] and the grey line is a Poisson distribution with mean 20. The simulations use Poisson distributions with different means depending on the simulation. 
The model assumes the sites exert forces on the cell location according to Hooke's law. The force is proportional to the distance. Thus, it is as if the sites are attached to the cell center (nucleus) with springs. In reality the mechanism is more complex, involving molecular motors contracting actin fibers.

There is a drag force on the cell center. The drag is modeled assuming the center is a sphere in a liquid with low Reynold's number and is proportional to the velocity of the center [26]. We denote the location of the cell center as $\mathbf{x}$, a point in $\mathbb{R}^{2}$. Likewise the location of each binding site is $\mathbf{s}_{j}$, a point in $\mathbb{R}^{2}$, where $j$ ranges from 1 to $K$.

The mathematical model uses Newton's second law of motion. Due to low Reynold's number the acceleration term can be ignored and the resulting equations are first order [26]. The equation of motion for the location of the cell is given by

$$
\mu \mathbf{x}^{\prime}=-\sum_{j=1}^{K} \alpha_{j}\left(\left\|\left(\mathbf{x}-\mathbf{s}_{j}\right)\right\|-\ell_{j}\right) \frac{\mathbf{x}-\mathbf{s}_{j}}{\left\|\mathbf{x}-\mathbf{s}_{j}\right\|} \psi_{j}(t)
$$

where $\mathbf{x}^{\prime}$ is the velocity of the cell center, $\ell_{j}$ is the length of the unstretched spring (taken to be $5 \mu m$, making the cell diameter at rest $10 \mu \mathrm{m}$, a typical size for rounded Dd cells), $\alpha_{j}$ is the spring constant, $\mathbf{s}_{j}$ denotes the location of the attached site, $\mu=0.101 \mathrm{~g} / \mathrm{h}$ is the drag coefficient, and $\psi_{j}(t)$ is a random variable which takes the values of 0 or 1 indicating that the site is unbound or bound respectively. The drag coefficient was determined using Stokes's drag assuming a 3 micron diameter sphere (the nucleus which typically ranges from 2-10 microns) in water.

Let $t_{p, j}$ be the most recent time adhesion site $j$ becomes bound, i.e., that $\psi_{j}$ made the transition from 0 to 1 , and $w_{p, j}$ is the binding duration time (averaging the $w_{p, j}$ gives the random attach time), i.e., that $\psi_{j}$ remains 1 for the most recent transition. Then

$$
\mathbf{s}_{j}(t)=\left\{\begin{array}{cl}
\mathbf{x}\left(t_{p, j}\right)+\mathbf{b}_{j} & \text { for } t_{p, j}<t<t_{p, j}+w_{p, j} \text { and } \psi_{j} \neq 0 \\
0 & \text { otherwise }
\end{array} .\right.
$$

The above equation says that the adhesion site is attached a distance $\left\|\mathbf{b}_{j}\right\|$ from wherever the center of the cell is located when the adhesion site attaches. It then remains at that position until the adhesion site detaches. In the simulations $\mathbf{b}_{j}$ is also a random variable where the length comes from a uniform distribution with range arbitrarily chosen from $10 \mu m$ to $15 \mu m$ in length which gives reasonable cell sizes. The direction of the vector is chosen from a uniform distribution from $-\frac{\pi}{2}$ to $\frac{\pi}{2}$ with a $60 \%$ chance and a $40 \%$ chance that the angle is in the range $\frac{\pi}{2}$ to $\frac{3 \pi}{2}$. This causes the cell to move in the forward $x$ direction but on average to not move in the $y$ direction.

\section{Results}

The cell configuration is shown at two different times for a typical simulation in figure 3 . The square markers show the location of the attached adhesion sites and the vectors originating at the squares show the force of the adhesion site on the substrate. The center of the cell is indiciated with a dot and the vector starting at that location shows the overall force vector on the center of the cell. Frequently the largest vectors are in opposition to the forward motion of the cell. This is consistent with measured forces in Dd [31]. For the standard setup the cell has a maximum of 10 adhesion sites that are randomly placed in an annulus centered at the nucleus. The inner radius would correspond to a rounded up cell and the outer radius is the farthest the adhesion sites can be extended to initially attach to the substrate. The adhesion sites remained attached for a random period of time and remain detached for a random period of time. The mean attachment time for actin foci in Dd has been measured at 20 seconds [30]. For the simulations the random variables have a Poisson distribution with different means (see figure 2). The default mean attachment time is 20 seconds and 25 seconds is the mean detach time.

The force a cell exerts is determined by the parameter $\alpha_{j}$ (the spring constant for the adhesion sites). Unless otherwise noted $\alpha_{j}=.206 \mathrm{nN} / \mu \mathrm{m}$. This was set so the force per adhesion site averaged over one minute intervals for the standard simulation described above is about 1 nanoNewton. The average force measured per actin foci for Dd assuming 5-6 foci per cell is about $1 \mathrm{nN}$ [32] the experimentally measured number of actin foci ranges from 3 to 9 [30].

\subsection{Attach Time and Detach Time}

In the first set of simulations the mean attachment time was varied from 10 to 70 seconds and the mean detach time was varied from 0 to 50 seconds. Recall that the binding sites attach randomly depending on the value of $\psi_{j}$. The random variable is determined 

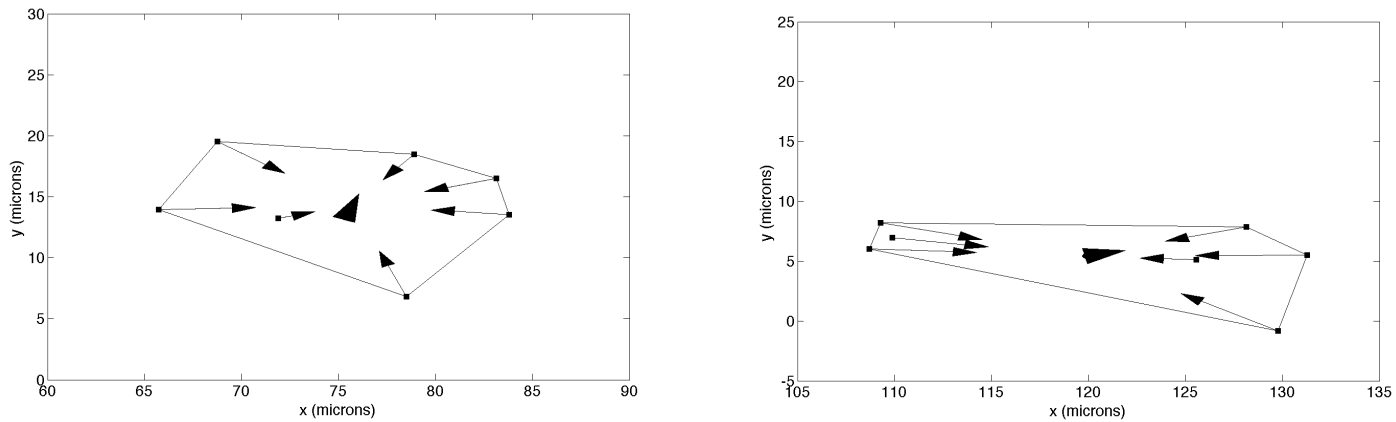

FIGURE 3. The cell configuration for a typical simulation is shown at time 0.0501 and 0.1663 hours. The large vector is the overall force on the cell center. The squares are the attachment points for the binding sites and the smaller vectors show the forces on the substrate due to the attachment sites. The cell is moving in the positive $x$ direction. Compare with figures 1-5 in [31].
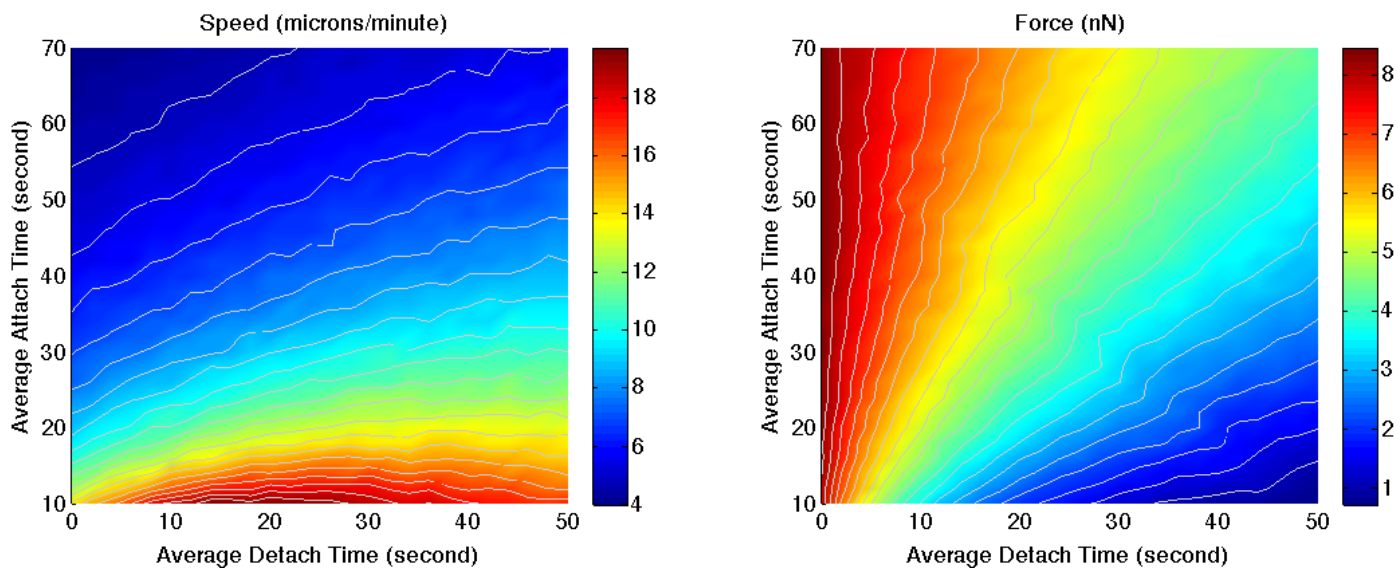

FIGURE 4. In the left panel the speed of a single cell is plotted against the mean attach time and mean detach time. In the right panel the time average of the sum of the absolute value of the forces is shown. The contour lines are plotted over the shading. The plot shows the average of 50 runs for each data point. The speed was calculated by averaging the average speed of the cell per minute over the duration of the simulation. The spring constants were all $\alpha_{j}=.206 \mathrm{nN} / \mu \mathrm{m}$, with a maximum of 10 adhesion sites.

by the distributions of the attach and detach times. The results can be seen in figure 4 where the average cell speed and cell force of 50 simulations (with different realizations of the random processes) for each data point are plotted. It is important to note that the cell velocity is not very sensitive to the detach time and depends heavily on the attachment time. The force changes from being more sensitive to detach time for low detach time values to less sensitive at high detach times. The sensitivity to attach time is opposite with low sensitivity at low detach times and higher sensitivity at high detach times.

These results are consistent with the model formulation and give insight into how cells move. When adhesion sites do not remain attached for very long the cell moves faster. When they remain attached for longer periods of time they end up in the back of the cell and inhibit the forward velocity. Since the detach time is not important to the cell velocity it suggests that most of the cell motion takes place soon after attachment. In our mathematical model this is true since the forces are proportional to the distance of the adhesion site from the cell center. This distance is greatest soon after attachment occurs or if the adhesion site remains attached for a longer period. The force plot confirms this idea.

At low detach times an adhesion site is typically free to attach, so most of the adhesion sites will be attached. This means that if the attach time is low then the force is relatively high (lower left corner of the left panel in figure 4) because the average distance from the cell center to the adhesion site is about the initial extension length. As the attach time increases, (moving to the top on the left side of the left panel in figure 4) the distance increases with the more distant adhesion sites at the back of the cell, inhibiting the motion but generating large forces opposed to the forward forces. As the detach time becomes larger fewer adhesion sites on average are attached to the substrate, particularly when the attach time is low (the lower right corner of the left panel in figure 4) and the overall force is reduced. 

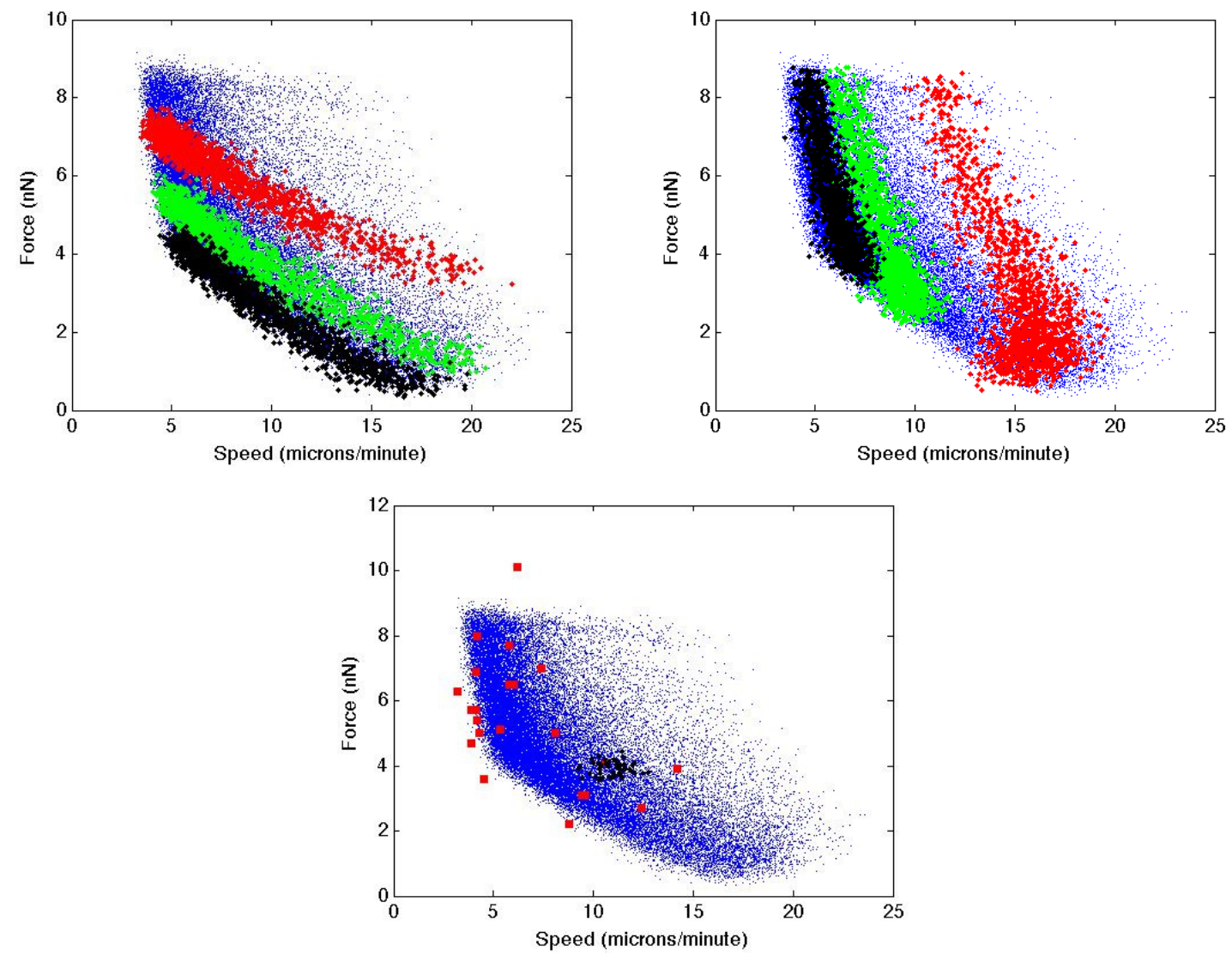

FIGURE 5. The force is plotted against the speed as blue dots for 40300 simulations of a single cell on a 2 dimensional substrate where the mean attach time is varied from 10 to 70 seconds, the mean detach time is varied from 0 to 50 seconds, and for each set of values 50 different simulations were run. In the left panel red, green, and black indicate simulations with a fixed average detach time of $10 \mathrm{~s}, 30 \mathrm{~s}$, and $50 \mathrm{~s}$ respectively. In the right panel red, green, and black indicate simulations with a fixed average attach time of $14 \mathrm{~s}, 34 \mathrm{~s}$, and 54 s respectively. The bottom panel shows the simulations in black asterisks where the average detach time is $24 \mathrm{~s}$ and the average attach time is 20 seconds. The red squares are experimentally measured values for individual Dd cells taken from [31]. Figure 4 was constructed from this data.

As the attach time increases, but the detach time remains large (moving from the right lower corner to the right upper corner of the left panel in figure 4) the force each adhesion site generates is increased thus increasing the overall force, yet remaining significantly less than when the detach time is low, since there are fewer attachments.

The cell velocity in all these simulations remains in biologically realistic ranges for Dd (see figure 5). The experimentally measured speed was determined by taking the average speed over increments of time between 18 and 20 seconds [31]. The speed was calculated in the simulations by averaging over a 60 second time interval.

Figure 5 shows plots for the cell velocity and the cell force for all the simulations. The speeds and forces compare well with the experimental data for Dd [31] which is also shown. In figure 5 the data points are shown for different parameter values. It is clear that for a fixed mean attach time there is a large variation in the forces and a small variation in the cell speed. As the mean attach time is increased the speeds decrease and the forces increase. For fixed mean detach times there is a large variation in the cell speed and a smaller but still significant variation in the cell force. As the mean detach time increases, the forces decrease and the speeds stay in the same range.

\subsection{Different Cell Strengths}

The velocity of the cell is not very sensitive to the force the cell exerts (i.e. the spring constant) as shown in figure 6, although the overall force on the substrate has a strong dependency. This is explained by considering all the adhesion site attachments. The 

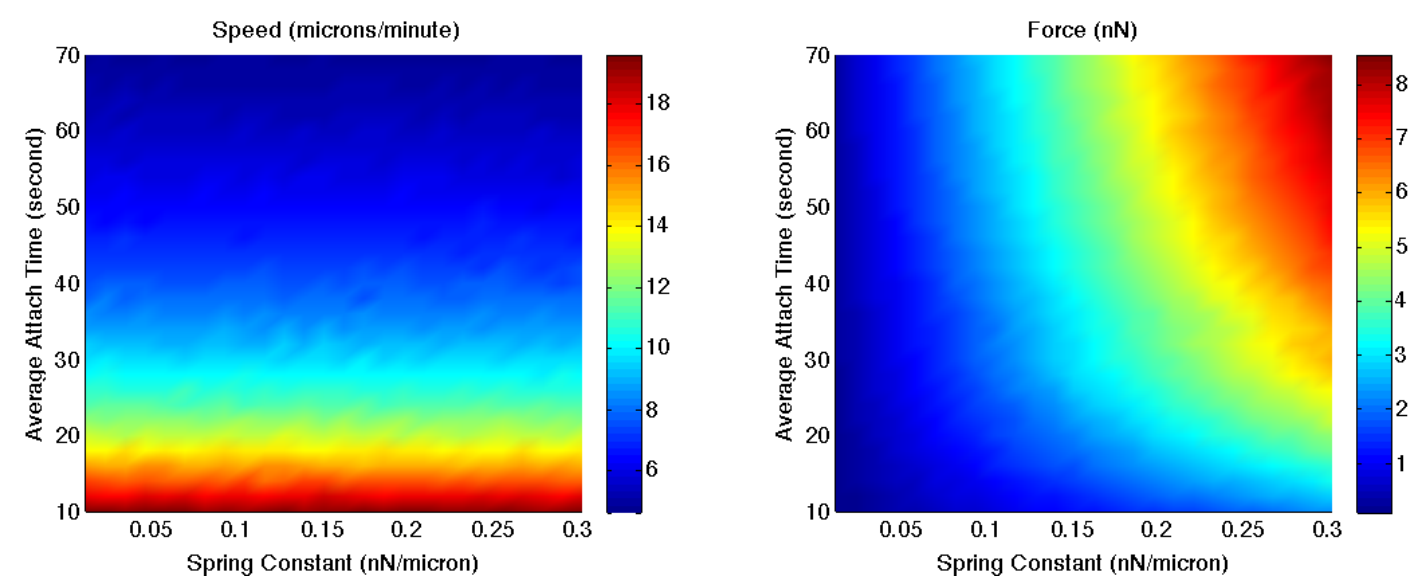

FIGURE 6. In the left panel the speed of a single cell is plotted against the mean attach time and strength of the cell. The cell speed is remarkably constant with respect to the strength of the cell. In the right panel the time average of the sum of the absolute value of the forces is shown. The plot shows the average of 50 runs for each data point. The mean detach time is 25 seconds.

attachments at the back of the cell oppose the forward motion whereas the attachments at the front of the cell encourage forward motion. When the forces on the binding sites are increased the overall force on the substrate increases. Since it appears that most of the cell motion occurs soon after attachment and detachment the instantaneous velocity of the cell increases but the average velocity does not. The cell moves quickly to a new position and then remains stationary until an adhesion site attaches or detaches. So, although the velocity is proportional to force, the average velocity does not change as the cell forces become higher. By making several simplifying assumptions an argument can be made (see the appendix) that the cell speed is approximately independent of $\alpha$, the spring constant (assuming all the spring constants are the same), provided that $\frac{\alpha \sum_{j} E\left[\psi_{j}\right]}{\mu} \Delta t$ is large. In this system and any cellular system, $\mu$, the viscous term, will be small compared to the force of the cell. Thus the observation that the speed is constant should be true for cells which exert forces stronger than Dd like leukocytes and fibroblasts. This may be an artifact of the mathematical model since a Hookean law is used for the force.

Again the scatter plots are shown in figure 7. For constant mean attach times, there is a wide variation in the forces, and a small variation in the cell speed with the cell speed decreasing as the mean attach time increases and the range of forces increases. For a fixed cell strength the cell speed has a large range and the resultant cell force does not vary as much. As the cell strength is increased the spread in the cell speed decreases and the speed seem to decrease. The spread in the cell forces increases and cell forces increase.

Different cell types exert different forces and move at different speeds. Fibroblasts are reported to exert forces from $1 \mathrm{nN}$ to 2650 $\mathrm{nN}$. The forces are measured in three different ways. First, forces are determined by how much a cell deforms the substrate [33,34] which gives the highest forces. Second, how cells deform micromachined devices [35-37], and finally by measuring the overall force on fibroblast populated collagen lattices and dividing by the total number of cells to get the force per cell $[38,39]$. The last two types of measurements are in the range of $0.1 \mathrm{nN}$ to $138 \mathrm{nN}$. Fibroblasts, when these forces are measured, are either stationary or moving up to $.7 \mu \mathrm{m}$ per minute [40,41]. Endothelial cells exert forces from $31-57 \mathrm{nN}$ with speeds reported from $.53-2.11 \mu \mathrm{m}$ per minute [35]. Murine dendritic cells moved at speeds of $2.3 \mu \mathrm{m}$ per minute with forces ranging from $6-32 \mathrm{nN}$ [42]. Finally, neutrophils move at speeds around $3.5 \mu \mathrm{m}$ per minute and show forces of $20-100 \mathrm{nN}$ depending on the stiffness of the substrate [43,44].

Figure 8 shows boxes which roughly show the velocity and force range of the various cell types and the scatter plots show results from the simulations with parameter values chosen to mimic the different cell types. By understanding the affect of mean attachment time on speed it was relatively easy to shift the data into the different regions. The parameter ranges for the different cell types are given in table 1. It appears the model would easily mimic speed and velocity relationships for various cell types.

\subsection{Varying Total Number of Binding Sites}

In the next set of simulations the effect of varying the total possible number of attachment sites available was explored. As expected, it did not differ much from the variation of the cell strength. The force plot shown in figure 9 is similar to the force plot for cell strength and is consistent with the force plot which varies with mean detach time. The correlation between force and mean detach time is opposite that of force and number of attachment sites. For long mean detach time there are fewer binding sites available to attach at any time since they have to wait before they can attach. The speed plot also looks similar but is not constant as with the cell strength. The fastest 

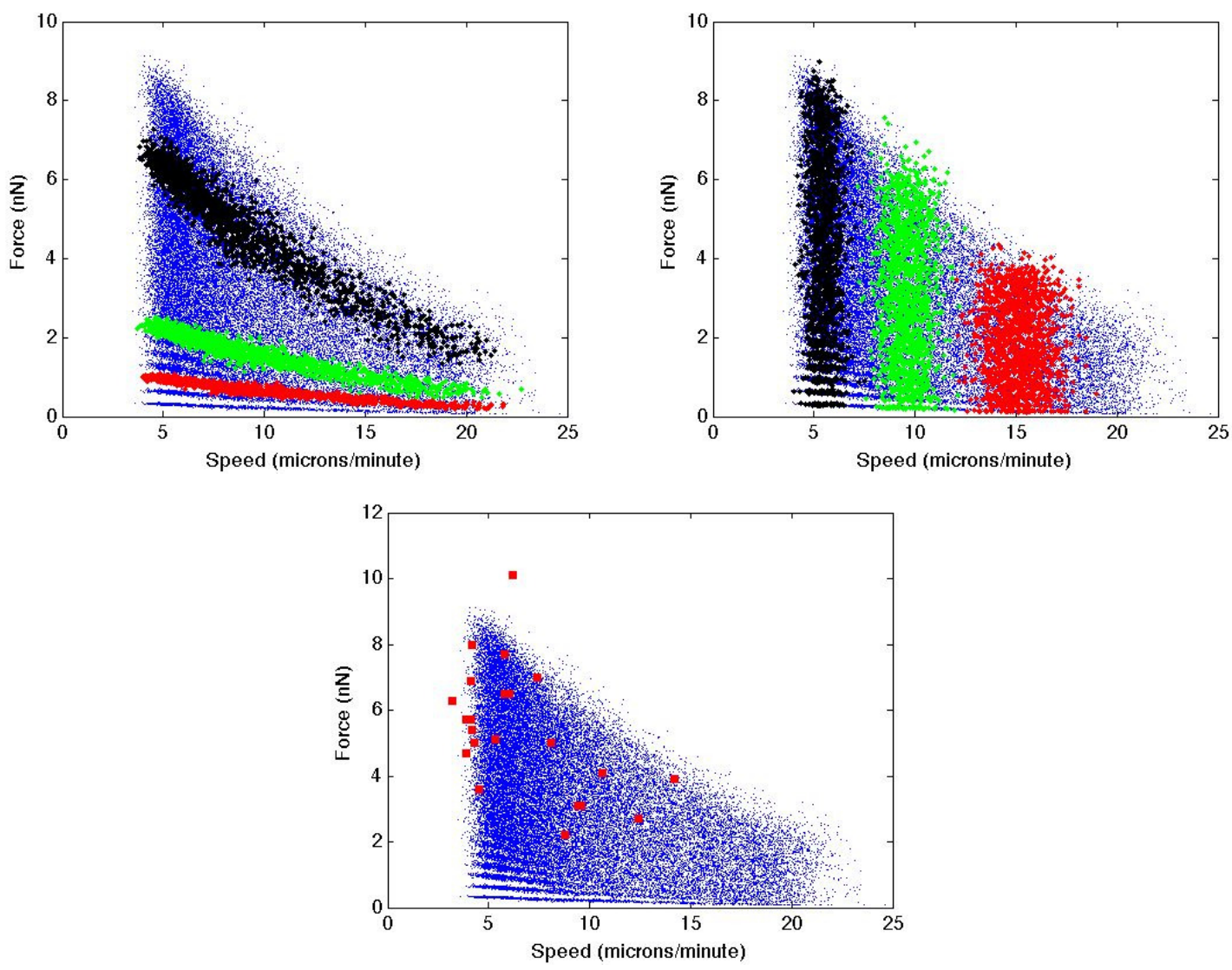

FIGURE 7. In the left panel red, green, and black indicate simulations with a fixed value for the spring constant where $\alpha_{j}$ is $0.0347,0.081018$, and $0.231 \mathrm{nN}$ per micron, respectively. In the right panel red, green, and black indicate simulations with a fixed average attach time of $16 \mathrm{~s}, 30 \mathrm{~s}$, and 60 $\mathrm{s}$ respectively. The force is plotted against the speed as blue dots for 40300 simulations of a single cell on a 2 dimensional substrate where the mean attach time is varied from 10 to 70 seconds, the mean detach time is varied from 0 to 50 seconds, and for each set of values 50 different simulations were run. Figure 6 was constructed from this data. The red squares are experimentally measured values for individual Dd cells taken from [31].

speeds occur at 10 attachment sites. This peak in speed is somewhat suprising. It is interesting to note that Dd has between 3-9 actin foci at any one time. This puts it in the range where speeds would be optimized. As the number of binding sites increase, the speed of the cell decreases for fixed mean attachment times. The data points used to construct the surface plots in figure 9 are plotted in figure 10 . The different stripes correspond to data when the number of attachment sites is different. As the number of attachment sites increases the same sort of distribution is seen as when the cell strength is increased. The distributions for a fixed number of binding sites are more curved than the distribution of points for fixed cell strength.

\subsection{New Force Rule}

With all the simulations shown, the strength of the attachment sites is the greatest when it is the farthest from the nucleus, which typically happens when the binding site first attaches or just before it releases (see figure 11). Biologically one might expect that the binding site strength is weak when it first attaches and increases with the age of the site as the cell reinforces the attachment and the cytoskeletal structure. Then after some period the strength of the site may begin to weaken as the cell dissassembles the site or prepares to release it. To test the model with more realistic adhesion site strength, simulations were run where the site strength was a function of bound duration as well as distance from the cell center. The new rule is the same as before only the strength of the attachment site is 


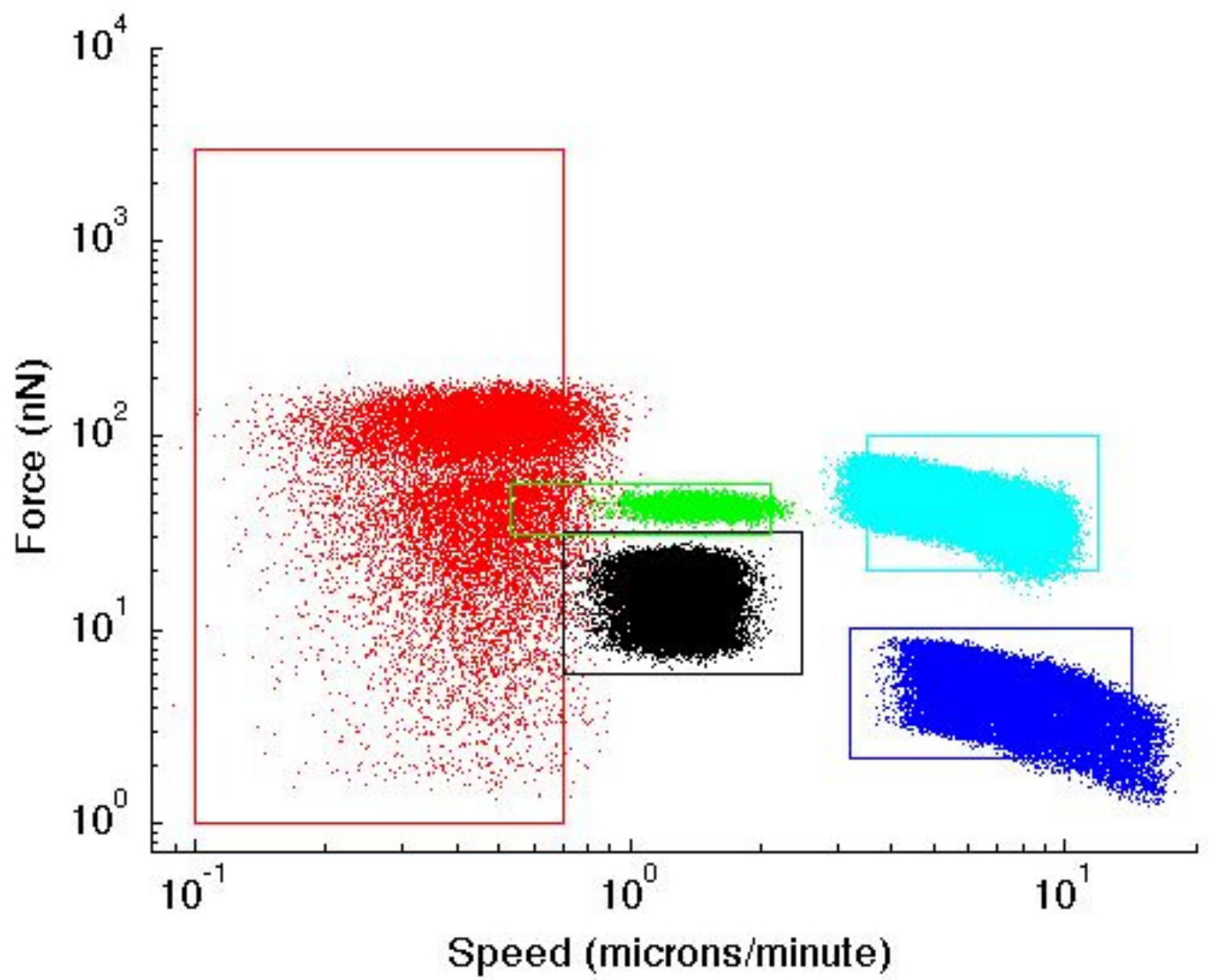

FIGURE 8. The boxes roughly outline the regions where experimental data has been reported for different cells types. The scatter plots are simulation results with parameters used to mimic the behavior of the different cells. Red denotes fibroblasts, black denotes murine dendritic cells, cyan denotes neutrophils, green denotes endothelial cells, and blue denotes Dd cells.
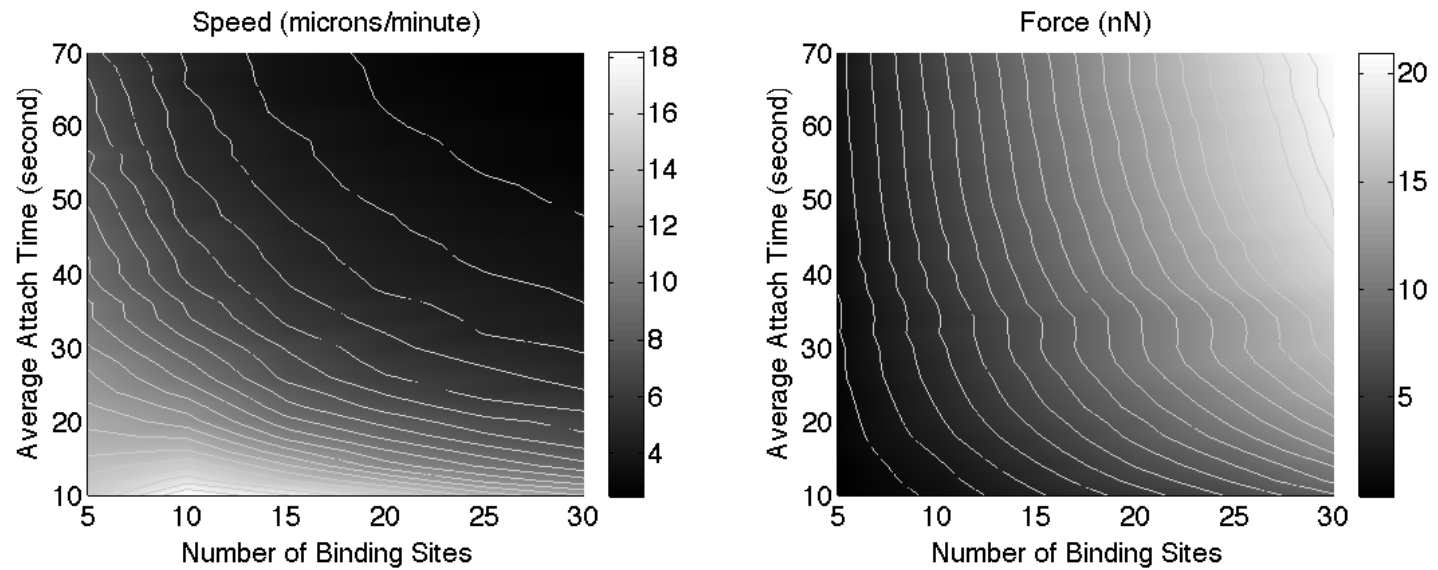

FIGURE 9. In the left panel the speed of a single cell is plotted against the mean attach time and total possible number of attachment sites. In the right panel the time average of the sum of the absolute value of the forces is shown. The contour lines are plotted over the shading. The plot shows the average of 50 runs for each data point. Mean detach time is 25 seconds. 
TABLE 1. Parameter ranges for different cell types

\begin{tabular}{lcc}
\hline \hline cell type & $\begin{array}{c}\text { range for } \\
\alpha_{j}(\mathrm{nN} / \mu \mathrm{m})\end{array}$ & $\begin{array}{c}\text { range for } \\
\text { mean attach time (s) }\end{array}$ \\
\hline fibroblast & $0.232-4.44$ & $810-1210$ \\
neutrophils & $1.17-2.33$ & $28-88$ \\
murine dendritic & $0.233-0.614$ & $230-408$ \\
endothelial & $1.17-1.18$ & $206-408$ \\
Dd & $0.116-0.348$ & $18-70$ \\
\hline
\end{tabular}

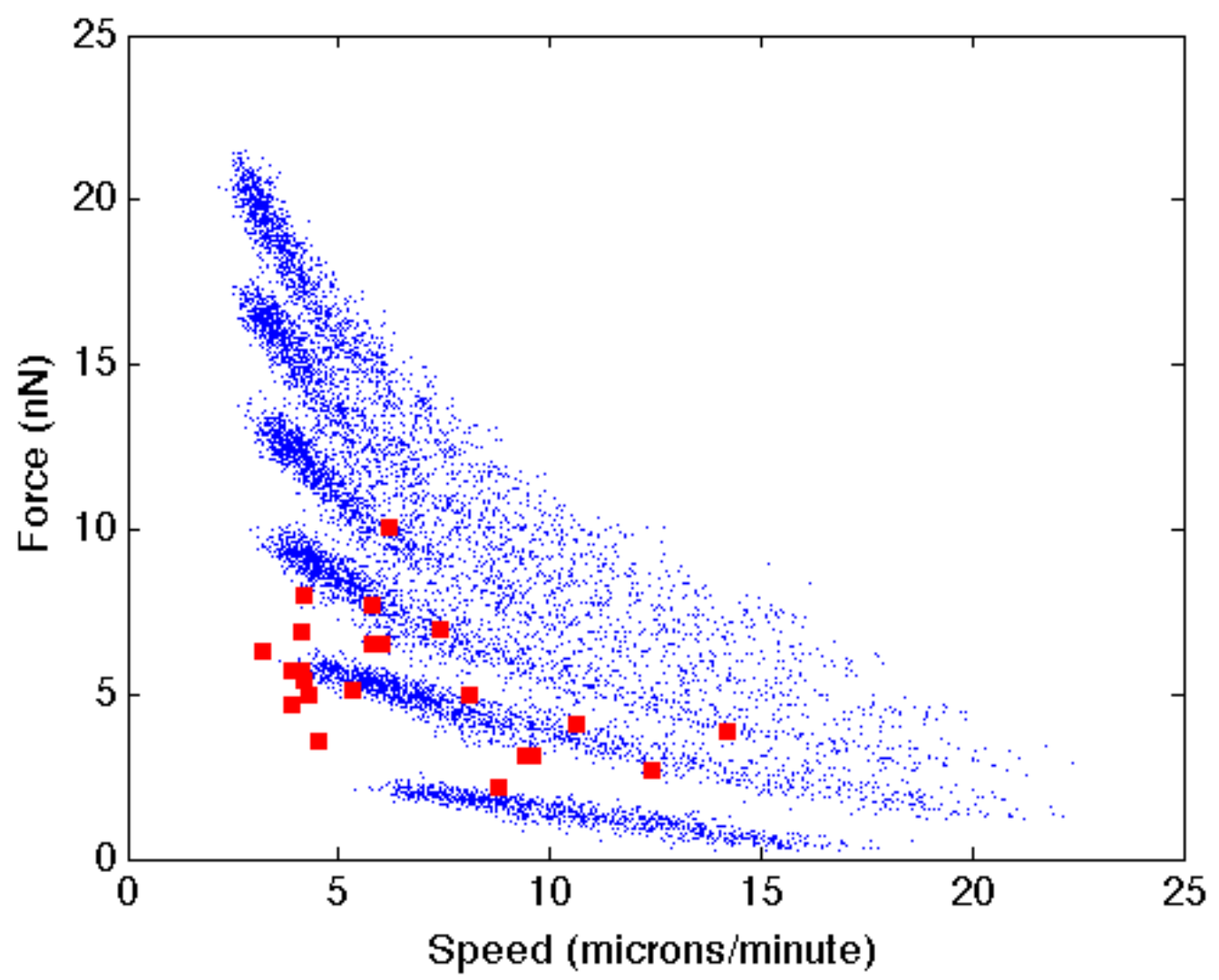

FIGURE 10. The panel shows the simulations where the average detach time is $24 \mathrm{~s}$ and the total number of possible binding sites ranges from 5 to 30 and the average attach time ranges from 10 to 70 . The data plotted was used to construct figure 9 . The squares are experimentally measured values for individual Dd cells taken from [31].

multiplied by a linear function which depends on the time the binding site has been attached. The new equation of motion is

$$
\mu \mathbf{x}^{\prime}=-\sum_{j=1}^{K} \alpha_{j}\left(\left\|\left(\mathbf{x}-\mathbf{s}_{j}\right)\right\|-\ell_{j}\right) \gamma_{j}\left(t-t_{p, j}\right) \frac{\mathbf{x}-\mathbf{s}_{j}}{\left\|\mathbf{x}-\mathbf{s}_{j}\right\|} \psi_{j}(t)
$$



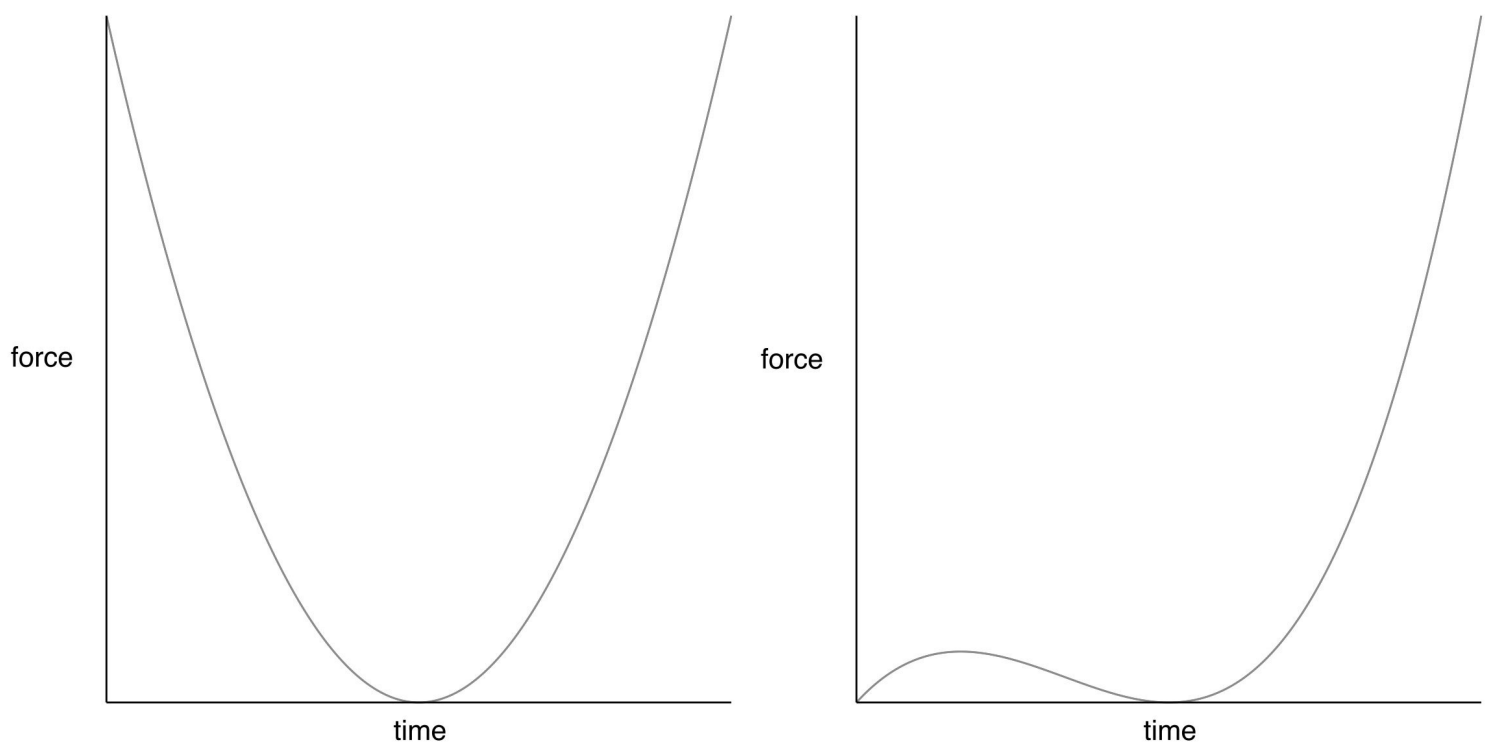

FIGURE 11. A quadratic curve is shown in the left panel which represents the force of an attachment site plotted against the time the site has been bound when the force is modeled as a spring. The right panel shows a representative curve of the new more realistic force rule which includes a term that strengthens the attachment site force with time.
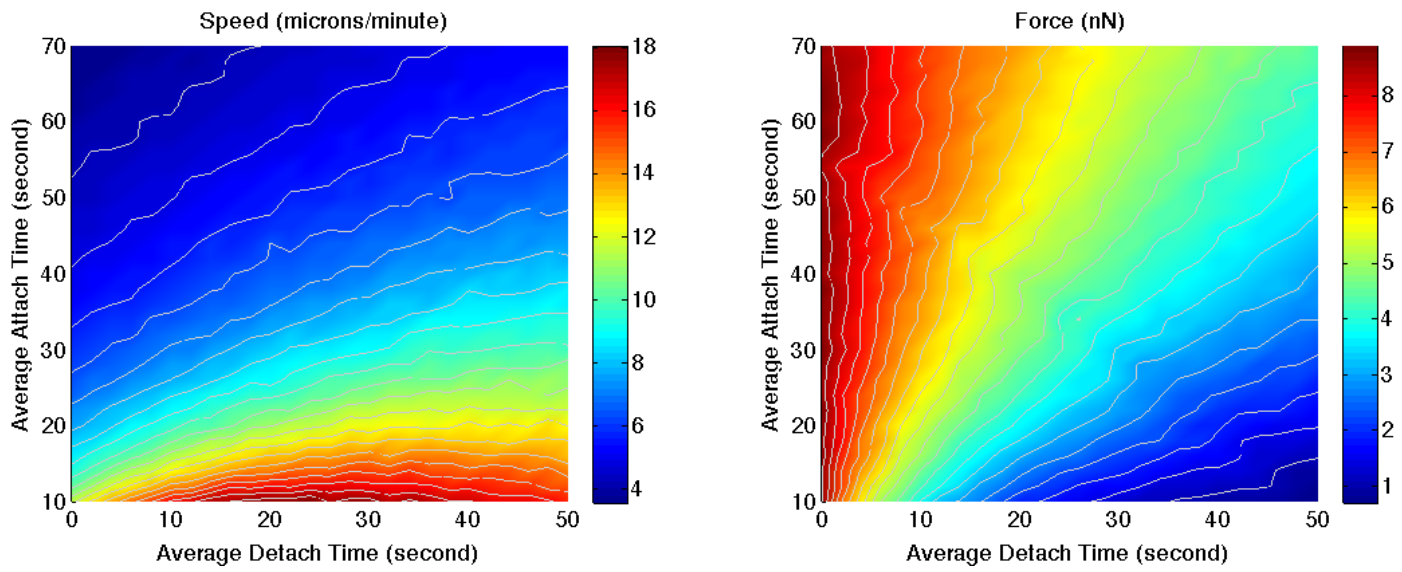

FIGURE 12. In the left panel the speed of a single cell is plotted against the mean attach time and mean detach time for simulations with the new force rule which depends on the time the attachment site has been bound. In the right panel the time average of the sum of the absolute value of the forces is shown. The contour lines are plotted over the shading. The plot shows the average of 50 runs for each data point. The speed was calculated by averaging the average speed of the cell per minute over the duration of the simulation which was 1 hour. The spring constant was $\alpha_{j}=.206 \mathrm{nN} / \mu \mathrm{m}$, with a maximum of 10 attachment sites.

Figure 11 shows qualitative curves which should represent the strength of a typical site as the attachment time varies.

Simulations with the new force rule gave results which did not differ significantly from the results with the old force rule (see figure 12). Because the new force rule has lower forces when the site initially attaches and the previous results indicated that the cell gets most of its forward motion from the initial attachment, it was suprising that none of the plots varied significantly. Examining the total change in the $x$ and $y$ position of the cell a difference in the two rules for generating forces is seen. In the first rule where the force depends only on the distance between the binding site and the cell center the average change in the $x$ coordinate is greater than in the second rule where the force also depends on the length of time the attachment site has been bound (see figure 13). There is no average change in the $y$ coordinate since there is no propensity to move in the $y$ direction. 

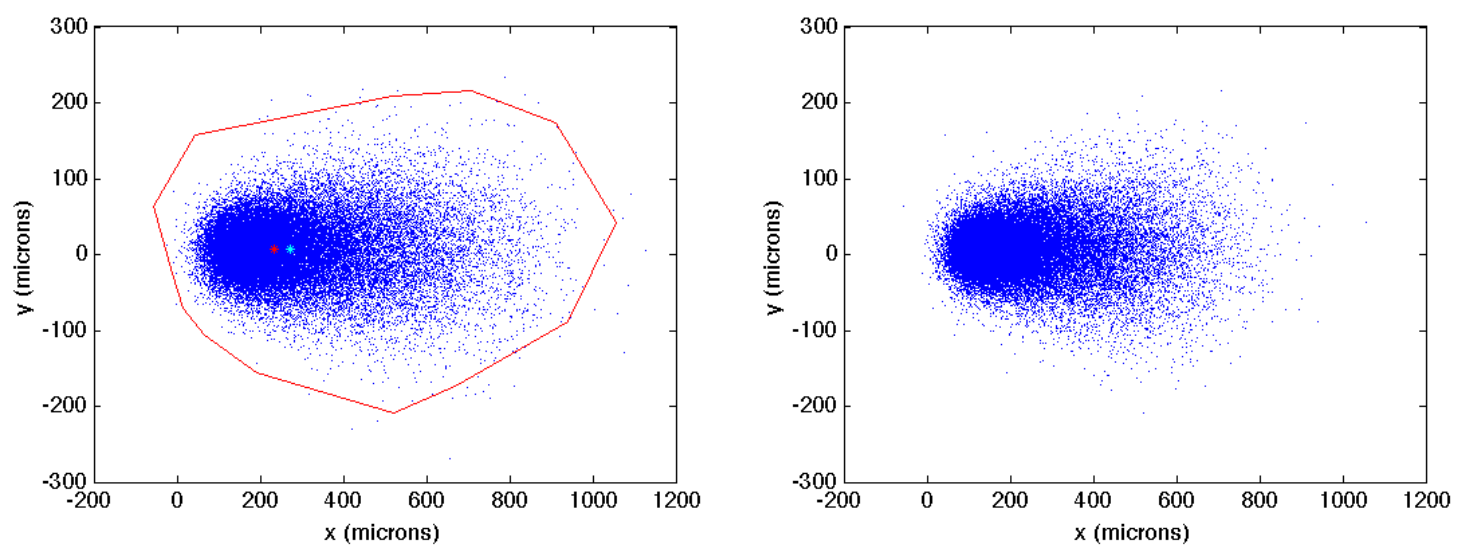

FIGURE 13. The total change in the $x$ coordinate and the $y$ coordinate is plotted for simulations using the first force rule (left panel) and simulations using the new force rule (right panel). The data for the old force rule is taken from the same simulations that are shown in figure 4 . The only difference between the two sets of data is the rule for generating force. The polygon shown in the left panel is the convex hull of the points shown in the right panel. The average change in $x$ and in $y$ for each data set is plotted in the left panel with the * indicating the new force rule and the square indicating the old force rule.

\section{Discussion}

This model asserts an important idea. The speed of the cell is mainly influenced by the mean attachment time. The mean detach time is not as significant which is comforting since it is a non-physical parameter. The amount of force the cell can transfer to the substrate is less important. This has been known for some time since for different cell types higher forces do not typically result in faster migrations.

The model further reveals that cell speed, when measured by averaging over time intervals will typically remain constant with respect to cell force. This reinforces the first point which is that the binding dynamics of the attachment sites are the most important factor determining speed. Cells strength will only matter as it affects the mechanosensing of the cell.

These simple simulations resulted in other interesting insights. When the binding sites remain attached for too long they inhibit cell motion and cause greater forces, but since they are in different directions the cell speed does not change much. As the mean attachment time is decreased, the binding site attachments are more effective at moving the cell and there is less overall force. Further, it seems that the specific details of the time dependence of the force do not affect these results but they can affect the efficiency of directed cell motion.

It is interesting to note that as the number of available attachment sites increases, the cell speed increases to a maximum and then decreases. This is similar to experimentally reported on integrin-ligand binding concentrations [45] where it seemed that speed increased as the cell gained more traction but would then decrease when there was too much traction. It is interesting to note that for Dd, a fast moving cell, the average number of actin foci is in the correct range to optimize the cell speed.

The two main conclusions suggested by the model simulations are the importance of the duration of binding site attachment for cell speed and the relative unimportance of the cell strength with regards to cell speed. The model also shows that detach times and the number of binding sites are much less important to cell motion, a point that gives confidence in the predictive power of the formulation. This work suggests that possible manipulation of cell motion rests on a greater understanding of the dynamics of cell attachments and that enterprise may best be forwarded by an understanding of the mechanisms controlling binding site attachment frequency.

\section{Appendix}

\subsection{Average Speed Independent of spring constant}

To understand why the velocity plots above seem to be independent of the spring constant, consider equation 1 where $\ell_{j}=0$, the rest length of the springs is zero, $\alpha_{j}=\alpha$, all the spring constants are the same, and $\mathbf{x}(0)=\mathbf{x}_{\mathbf{0}}$ gives the initial condition. We will further assume that the expected value of $\mathbf{s}_{j}$ and $\psi_{j}$ are independent of $\mathbf{x}$. Equation 1 becomes

$$
\mu \mathbf{x}^{\prime}=-\sum_{j=1}^{K} \alpha\left(\mathbf{x}-\mathbf{s}_{j}\right) \psi_{j}(t)
$$


Let $E[x]$ denote the expectated value of $x$. Taking the expectation of equation 4 gives

$$
\mu E[\mathbf{x}]^{\prime}=-\sum_{j=1}^{K} \alpha\left(E[\mathbf{x}] E\left[\psi_{j}\right]-E\left[\mathbf{s}_{j}\right] E\left[\psi_{j}\right]\right)
$$

Let $E\left[\psi_{j}\right]=\beta_{j}$, then

$$
E[\mathbf{x}]=\lambda \mathbf{m}+\left(\mathbf{x}_{0}-\alpha M\right) e^{-\frac{\alpha}{\lambda} t}
$$

where $\mathbf{m}=\sum \beta_{j} E\left[\mathbf{s}_{j}\right]$ and $\lambda=\frac{\mu}{\sum \beta_{j}}$. Thus the average velocity over a time period $\Delta t$ starting at $t=0$ is

$$
\frac{E[\mathbf{x}(\Delta t)]-\mathbf{x}_{0}}{\Delta t}=\frac{t}{\Delta t}\left(\lambda \mathbf{m}-\mathbf{x}_{0}\right)\left(1-e^{-\frac{\alpha}{\lambda} \Delta t}\right) .
$$

If $\frac{\alpha}{\lambda} \Delta t$ is large so the exponential is close to zero the average speed will not depend on $\alpha$. Larger spring constants do not change the result.

\section{REFERENCES}

[1] Sonnemann, K. J., and Bement, W. M., 2011. "Wound repair: Toward understanding and integration of single-cell and multicellular wound responses". Annual Review of Cell and Developmental Biology, Vol 27, 27, pp. 237-263.

[2] Krawczyk, W., 1971. "A pattern of epidermal cell migration during wound healing". The Journal of cell biology, 49(2), pp. 247263.

[3] Tanner, K., Ferris, D., Lanzano, L., Mandefro, B., Mantulin, W., Gardiner, D., Rugg, E., and Gratton, E., 2009. "Coherent movement of cell layers during wound healing by image correlation spectroscopy". Biophysical journal, 97(7), pp. 2098-2106.

[4] Friedl, P., and Wolf, K., 2003. "Tumour-cell invasion and migration: Diversity and escape mechanisms". Nature Reviews Cancer, 3(5), May, pp. 362-374.

[5] Yilmaz, M., and Christofori, G., 2010. "Mechanisms of motility in metastasizing cells". Molecular Cancer Research, 8(5), p. 629.

[6] Keller, R., Davidson, L., Edlund, A., Elul, T., Ezin, M., Shook, D., and Skoglund, P., 2000. "Mechanisms of convergence and extension by cell intercalation". Philosophical Transactions of the Royal Society of London. Series B: Biological Sciences, 355(1399), pp. 897-922.

[7] Mammoto, T., and Ingber, D., 2010. "Mechanical control of tissue and organ development". Development, 137(9), pp. 1407-1420.

[8] Rieu, J.-P., Saito, T., Delanoë-Ayari, H., Sawada, Y., and Kay, R. R., 2009. "Migration of dictyostelium slugs: anterior-like cells may provide the motive force for the prespore zone". Cell Motil Cytoskeleton, 66(12), Dec, pp. 1073-86.

[9] Holle, A. W., and Engler, A. J., 2011. "More than a feeling: discovering, understanding, and influencing mechanosensing pathways". Current Opinion In Biotechnology, 22(5), Oct., pp. 648-654.

[10] Huttenlocher, A., and Horwitz, A. R., 2011. "Integrins in cell migration”. Cold Spring Harb Perspect Biol, 3(9), Sep, p. a005074.

[11] Izzard, C., and Lochner, L., 1976. "Cell-to-substrate contacts in living fibroblasts: an interference reflexion study with an evaluation of the technique". Journal of cell science, 21(1), pp. 129-159.

[12] Heath, J., and Dunn, G., 1978. "Cell to substratum contacts of chick fibroblasts and their relation to the microfilament system. a correlated interference-reflexion and high-voltage electron-microscope study". Journal of cell science, 29(1), pp. 197-212.

[13] Lazarides, E., and Burridge, K., 1975. "r,-actinin: immunofluorescent localization of a muscle structural protein in nonmuscle cells". Cell, 6, pp. 289-298.

[14] Gardel, M. L., Schneider, I. C., Aratyn-Schaus, Y., and Waterman, C. M., 2010. "Mechanical integration of actin and adhesion dynamics in cell migration". Annu Rev Cell Dev Biol, 26, Nov, pp. 315-33.

[15] Lauffenburger, D., and Horwitz, A., 1996. "Cell migration: A physically integrated molecular process". Cell, 84(3), Feb., pp. 359-369.

[16] Dickinson, R., 2000. "A generalized transport model for biased cell migration in an anisotropic environment". Journal of Mathematical Biology, 40(2), Feb., pp. 97-135. 
[17] Sandersius, S. A., and Newman, T. J., 2008. "Modeling cell rheology with the subcellular element model". Physical Biology, 5(1), Mar., p. 015002.

[18] Keren, K., Pincus, Z., Allen, G. M., Barnhart, E. L., Marriott, G., Mogilner, A., and Theriot, J. A., 2008. "Mechanism of shape determination in motile cells". Nature, 453(7194), May, pp. 475-U1.

[19] Mogilner, A., and Oster, G., 2003. "Force generation by actin polymerization ii: The elastic ratchet and tethered filaments". Biophysical Journal, 84(3), Mar., pp. 1591-1605.

[20] Schreiber, C. H., Stewart, M., and Duke, T., 2010. "Simulation of cell motility that reproduces the force-velocity relationship". Proceedings of the National Academy of Sciences of the United States of America, 107(20), May, pp. 9141-9146.

[21] Paszek, M. J., Boettiger, D., Weaver, V. M., and Hammer, D. A., 2009. "Integrin clustering is driven by mechanical resistance from the glycocalyx and the substrate". Plos Computational Biology, 5(12), Dec., p. e1000604.

[22] Bottino, D., and Fauci, L., 1998. "A computational model of ameboid deformation and locomotion". European Biophysics Journal With Biophysics Letters, 27(5), pp. 532-539.

[23] Herant, M., and Dembo, M., 2010. "Form and function in cell motility: From fibroblasts to keratocytes". Biophysical Journal, 98(8), Apr., pp. 1408-1417.

[24] Buenemann, M., Levine, H., Rappel, W.-J., and Sander, L. M., 2010. "The role of cell contraction and adhesion in dictyostelium motility”. Biophysical Journal, 99(1), July, pp. 50-58.

[25] Ionides, E., Fang, K., Isseroff, R., and Oster, G., 2004. "Stochastic models for cell motion and taxis". Journal of Mathematical Biology, 48(1), Jan., pp. 23-37.

[26] Dallon, J. C., and Othmer, H. G., 2004. "How cellular movement determines the collective force generated by the Dictyostelium discoideum slug". J. Theor. Biol., 231, pp. 203-222.

[27] Mallet, D. G., and Pettet, G. J., 2006. "A mathematical model of integrin-mediated haptotactic cell migration". Bulletin of Mathematical Biology, 68(2), Feb., pp. 231-253.

[28] Ulrich, F., and Heisenberg, C.-P., 2009. "Trafficking and cell migration”. Traffic, 10(7), Jul, pp. 811-8.

[29] Gumbiner, B. M., 1996. "Cell adhesion: the molecular basis of tissue architecture and morphogenesis". Cell, 84(3), Feb, pp. 34557.

[30] Uchida, K., and Yumura, S., 2004. "Dynamics of novel feet of dictyostelium cells during migration". Journal of Cell Science, 117(8), Mar., pp. 1443-1455.

[31] Delanoë-Ayari, H., Iwaya, S., Maeda, Y. T., Inose, J., Rivière, C., Sano, M., and Rieu, J.-P., 2008. "Changes in the magnitude and distribution of forces at different dictyostelium developmental stages". Cell Motil Cytoskeleton, 65(4), Apr, pp. 314-31.

[32] Delanoë-Ayari, H., and Rieu, J. P., 2010. "4d traction force microscopy reveals asymmetric cortical forces in migrating dictyostelium cells"'. Phys. Rev. Lett.; Physical Review Letters, 105(24).

[33] Fray, T. R., Molloy, J. E., Armitage, M. P., and Sparrow, J. C., 1998. "Quantification of single human dermal fibroblast contraction". Tissue Eng, 4(3), pp. 281-91.

[34] Wrobel, L. K., Fray, T. R., Molloy, J. E., Adams, J. J., Armitage, M. P., and Sparrow, J. C., 2002. "Contractility of single human dermal myofibroblasts and fibroblasts". Cell Motility and the Cytoskeleton, 52, pp. 82-90.

[35] Tymchenko, N., Wallentin, J., Petronis, S., Bjursten, L. M., Kasemo, B., and Gold, J., 2007. "A novel cell force sensor for quantification of traction during cell spreading and contact guidance". Biophysical Journal, 93(1), July, pp. 335-345.

[36] Galbraith, C. G., and Sheetz, M. P., 1997. "A micromachined device provides a new bend on fibroblast traction forces". Proc Natl Acad Sci U S A, 94(17), Aug, pp. 9114-8.

[37] Munevar, S., Wang, Y., and Dembo, M., 2001. "Traction force microscopy of migrating normal and h-ras transformed 3t3 fibroblasts". Biophysical Journal, 80(4), Apr., pp. 1744-1757.

[38] Brown, R., Prajapati, R., McGrouther, D., Yannas, I., and Eastwood, M., 1998. "Tensional homeostasis in dermal fibroblasts: Mechanical responses to mechanical loading in three-dimensional substrates". Journal of cellular physiology, 175(3), pp. 323332.

[39] Delvoye, P., Wiliquet, P., Levêque, J. L., Nusgens, B. V., and Lapière, C. M., 1991. "Measurement of mechanical forces generated by skin fibroblasts embedded in a three-dimensional collagen gel". J Invest Dermatol, 97(5), Nov, pp. 898-902.

[40] Kaiser, J.-P., Reinmann, A., and Bruinink, A., 2006. "The effect of topographic characteristics on cell migration velocity". Biomaterials, 27(30), Oct., pp. 5230-5241.

[41] Nenasheva, T. A., Carter, T., and Mashanov, G. I., 2012. "Automatic tracking of individual migrating cells using low-magnification dark-field microscopy". Journal of Microscopy, 246(1), Apr., pp. 83-88.

[42] Ricart, B. G., Yang, M. T., Hunter, C. A., Chen, C. S., and Hammer, D. A., 2011. "Measuring traction forces of motile dendritic cells on micropost arrays". Biophysical Journal, 101(11), Dec., pp. 2620-2628. 
[43] Jannatt, R. A., Dembo, M., and Hammer, D. A., 2011. "Traction forces of neutrophils migrating on compliant substrates". Biophysical Journal, 101(3), Aug., pp. 575-584.

[44] Smith, L. A., Aranda-Espinoza, H., Haun, J. B., Dembo, M., and Hammer, D. A., 2007. "Neutrophil traction stresses are concentrated in the uropod during migration". Biophysical Journal, 92(7), Apr., pp. L58-L60.

[45] Palecek, S. P., Loftus, J. C., Ginsberg, M. H., Lauffenburger, D. A., and Horwitz, A. F., 1997. "Integrin-ligand binding properties govern cell migration speed through cell-substratum adhesiveness". Nature, 385(6616), Feb, pp. 537-40. 